\title{
AUTHOR INDEX VOLUME 9 (1999)
}

Adeodato, P.J.L., see de Souto, M.C.P.

9 (1999) 203-210

Angeleri, E., Apolloni, B., de Falco, D. \& Grandi, L., DNA fragment assembly using neural prediction techniques

Anlauf, J.K., see Grassmann, C.

Apolloni, B., see Angeleri, E.

Araújo, A.F.R., see Barreto, G.A.

Arif, M. \& Inooka, H., Human learning characteristics in the tracking tasks of iterative nature

Auda, G. \& Kamel, M., Modular neural networks: a survey

Barret, C., see Vigneron, V.

Barreto, G.A. \& Araújo, A.F.R., Unsupervised learning and recall of temporal sequences: an application to robotics

Benton, C., see McOwan, P.W.

Bernier, J.L., see Ros, E.

Bernier, J.L., Ortega, J., Ros, E., Rojas, I. \& Prieto, A., A new measurement of noise immunity and generalization ability for MLPs

Blanchard, M., Verschure, P.F.M.J. \& Rind, F.C., Using a mobile robot to study locust collision avoidance responses

Braga, A., see Nobre, C.

Braga, A.P. \& Ludermir, T.B., Editorial

Braga, A.P., see Parma, G.G.

Braga, J.L., see Nobre, C.

Breslin, C. \& Smith, L.S., Silicon cellular morphology

Brouwer, R.K., Growing of a fuzzy recurrent artificial neural network (FRANN) for pattern classification

Brouwer, R.K., The backpropagation algorithm in J, a fast prototyping tool for researching neural networks

Caminhas, W.M., see Landim, R.P.

Campbell, D.R., see Hussain, A.

Carlile, S., see van Schaik, A.

Cavaco, S. \& Hallam, J., A biologically plausible acoustic motion detection neural network

Cavalieri, S., Solving linear integer programming problems by a novel neural model

Chan, H.Y., Hui, S. \& Żak, S.H., Synchronous and asynchronous brain-state-in-a-box information system neural models

Chang, H.Y., see Fu, H.-C.

Chang, P.-R., Color image correction for scanner and printer using B-spline CMAC neural networks

Chapman, T. \& Webb, B., A neuromorphic hair sensor model of wind-mediated escape in the cricket

Chen, K. \& Chi, H., A modular neural network architecture for pattern classification based on different feature sets

Chi, H., see Chen, K.

Costa, J.A.F. \& Netto, M.L.A., Estimating the number of clusters in multivariate data by self-organizing maps

Dale, J., see McOwan, P.W.

de Carvalho, A., see Nobre, C.

de Falco, D., see Angeleri, E.

de Menezes, B.R., see Landim, R.P.

de Menezes, B.R., see Parma, G.G.

de Pinho, M., see Mazza, M.B.
9 (1999) 523-544

9 (1999) 473-478

9 (1999) 523-544

9 (1999) 235-242

9 (1999) 301-310

9 (1999) 129-151

9 (1999) 227-234

9 (1999) 235-242

9 (1999) 429-434

9 (1999) 485-490

9 (1999) 511-521

9 (1999) 405-410

9 (1999) 257-264

9 (1999) 163-165

9 (1999) 187-193

9 (1999) 257-264

9 (1999) 491-495

9 (1999) 335-350

9 (1999) 273-284

9 (1999) 211-217

9 (1999) 461-466

9 (1999) 441-446

9 (1999) 453-459

9 (1999) 27-39

9 (1999) 61-74

9 (1999) 545-561

9 (1999) 115-128

9 (1999) 397-403

9 (1999) 563-581

9 (1999) 563-581

9 (1999) 195-202

9 (1999) 429-434

9 (1999) 257-264

9 (1999) 523-544

9 (1999) 211-217

9 (1999) 187-193

9 (1999) 265-271 
de Souto, M.C.P., Adeodato, P.J.L. \& Ludermir, T.B., Sequential RAM-based neural networks: learnability, generalisation, knowledge extraction, and grammatical inference Dong, C., see Shi, D.

Douglas, R.J., see Mudra, R.

Edwards, R.T., Acoustic transient classification with a template correlation processor

El Khattabi, N., see Jedra, M.

Eriksson, B.E., see Smith, L.S.

Essaid, A., see Jedra, M.

Fechine, J.M., see Madeiro, F.

Fu, H.-C., Xu, Y.Y. \& Chang, H.Y., Recognition of handwritten similar Chinese characters by self-growing probabilistic decision-based neural network

Galvão, R.K.H. \& Yoneyama, T., Improving the discriminatory capabilities of a neural classifier by using a biased-wavelet layer

Giannakopoulos, X., Karhunen, J. \& Oja, E., An experimental comparison of neural algorithms for independent component analysis and blind separation

Gielen, S., see van de Laar, P.

Glover, M., see Smith, L.S.

Grandi, L., see Angeleri, E.

Grassmann, C. \& Anlauf, J.K., Fast digital simulation of spiking neural networks and neuromorphic integration with SPIKELAB

Hagiwara, M., see Osana, Y.

Hahnloser, R., see Mudra, R.

Hallam, J., see Cavaco, S.

Hamilton, A., see Smith, L.S.

Harrison, R.R. \& Koch, C., An analog VLSI implementation of a visual interneuron: Enhanced sensory processing through biophysical modeling

Hemerly, E.M., see Ribeiro, C.H.C.

Heskes, T., see van de Laar, P.

Honda, A., see Kameda, S.

Hui, S., see Chan, H.Y.

Hussain, A. \& Campbell, D.R., Speech-intelligibility improvements using a binaural adaptive-scheme based conceptually on human auditory processing

Hussain, A., Multi-sensor neural-network processing of noisy speech

Inooka, H., see Arif, M.

Jedra, M., El Khattabi, N., Limouri, M. \& Essaid, A., Recognition of seed varieties using a temporal organisation map analysis of electrophoretic images

Jin, C., see van Schaik, A.

Johnston, A., see McOwan, P.W.

Kameda, S., Honda, A. \& Yagi, T., Real time image processing with an analog vision chip system

Kamel, M., see Auda, G.

Karhunen, J., see Giannakopoulos, X.

Koch, C., see Harrison, R.R.

Landim, R.P., de Menezes, B.R., Silva, S.R. \& Caminhas, W.M., Nonlinear system identification using a neo fuzzy neuron algorithm: electrical drive application

Lehtokangas, M., Feedforward neural network with adaptive reference pattern layer

Li, C.-K., see Lin, C.-S.

Limouri, M., see Jedra, M.

Lin, C.-S. \& Li, C.-K., A memory-based self-generated basis function neural network

Lin, C.-T., Nein, H.-W. \& Lin, W.-C., A space-time delay neural network for motion recognition and its application to lipreading

Lin, W.-C., see Lin, C.-T.

Ludermir, T., see Nobre, C.

Ludermir, T.B., see Braga, A.P.

Ludermir, T.B., see de Souto, M.C.P.

Madeiro, F., Vilar, R.M., Fechine, J.M. \& Neto, B.G.A., A self-organizing algorithm for vector quantizer design applied to signal processing

Martineli, E., see Nobre, C.

Mazza, M.B., de Pinho, M. \& Roque, A.C., Biologically plausible models of topographic map formation in the somatosensory and auditory cortices
9 (1999) 203-210
9 (1999) 497-509
9 (1999) 411-416
9 (1999) 435-440
9 (1999) $153-162$
9 (1999) 479-484
9 (1999) 153-162
9 (1999) 219-226
9 (1999) 545-561
9 (1999) 167-174
9 (1999) 99-114
9 (1999) 75-85
9 (1999) 479-484
9 (1999) 523-544
9 (1999) 473-478
9 (1999) 285-299
9 (1999) 411-416
9 (1999) 453-459
9 (1999) 479-484
9 (1999) 391-395
9 (1999) 243-249
9 (1999) 75-85
9 (1999) 423-428
9 (1999) 61-74
9 (1999) 461-466
9 (1999) 467-471
9 (1999) 301-310
9 (1999) 153-162
9 (1999) 441-446
9 (1999) 429-434
9 (1999) 423-428
9 (1999) 129-151
9 (1999) 99-114
9 (1999) 391-395
9 (1999) 211-217
9 (1999) 1-9
9 (1999) 41-59
9 (1999) 153-162
9 (1999) 41-59
9 (1999) 311-334
9 (1999) 311-334
9 (1999) 257-264
9 (1999) 163-165
9 (1999) 203-210
9 (1999) 219-226
9 (1999) 257-264
9 (1999) 265-271 
McOwan, P.W., Benton, C., Dale, J. \& Johnston, A., A multi-differential neuromorphic approach to motion detection

Meyer-Bäse, A.,Asymptotic hyperstability of a class of neural networks

Möller, R., Visual homing in analog hardware

Monfroglio, A., Neural networks and linear programming for the satisfiability problem

Mudra, R., Hahnloser, R. \& Douglas, R.J., Integrating neuromorphic action-oriented perceptual inputs to generate a navigation behaviour for a robot

Nein, H.-W., see Lin, C.-T.

Neto, B.G., see Madeiro, F.

Netto, M.L.A., see Costa, J.A.F.

Nobre, C., Martineli, E., Braga, A., de Carvalho, A., Rezende, S., Braga, J.L. \& Ludermir, T., Knowledge extraction: a comparison between symbolic and connectionist methods

Oja, E., see Giannakopoulos, X.

Ortega, J., see Bernier, J.L.

Osana, Y. \& Hagiwara, M., Successive learning in hetero-associative memory using chaotic neural networks

Palomar, D., see Ros, E.

Parma, G.G., de Menezes, B.R. \& Braga, A.P., Neural networks learning with sliding mode control: the sliding mode backpropagation algorithm

Paschke, P., see Schauer, C.

Pedreira, C.E., see Pedroza, L.C.

Pedroza, L.C. \& Pedreira, C.E., Multilayer neural networks and function reconstruction by using a priori knowledge

Pelayo, F.J., see Ros, E.

Prieto, A., see Bernier, J.L.

Prieto, A., see Ros, E.

Pujari, A.K., see Rao, M.S.

Rao, M.S. \& Pujari, A.K., A new neural network architecture with associative memory, pruning and order-sensitive learning

Rezende, S., see Nobre, C.

Ribeiro, C.H.C. \& Hemerly, E.M., Autonomous learning based on cost assumptions: theoretical studies and experiments in robot control

Rind, F.C., see Blanchard, M.

Rojas, I., see Bernier, J.L.

Rojas, I., see Ros, E.

Roque, A.C., see Mazza, M.B.

Ros, E., see Bernier, J.L.

Ros, E., Pelayo, F.J., Palomar, D., Rojas, I., Bernier, J.L. \& Prieto, A., Stimulus correlation and adaptive motion detection using spiking neurons

Schauer, C. \& Paschke, P., A spike-based model of binaural sound localization

Shi, D., Dong, C. \& Yeung, D.S., Neocognitron's parameter tuning by genetic algorithms

Silva, S.R., see Landim, R.P.

Smith, L.S., see Breslin, C.

Smith, L.S., Eriksson, B.E., Hamilton, A. \& Glover, M., SPIKEII: an integrate-and-fire a VLSI chip

Szu, H., Thermodynamics energy for both supervised and unsupervised learning neural nets at a constant temperature

Tam, P.K.S., see Wu, A.

van de Laar, P., Heskes, T. \& Gielen, S., Partial retraining: a new approach to input relevance determination

van Schaik, A., Jin, C. \& Carlile, S., Human localisation of band-pass filtered noise

Verschure, P.F.M.J., see Blanchard, M.

Vigneron, V. \& Barret, C., Approximation techniques for neuromimetic calculus

Vilar, R.M., see Madeiro, F.

Webb, B., see Chapman, T.

Webb, B., A framework for models of biological behaviour

Wörgötter, F., An ASIC-Chip for stereoscopic depth analysis in video-real-time based on visual cortical cell behavior

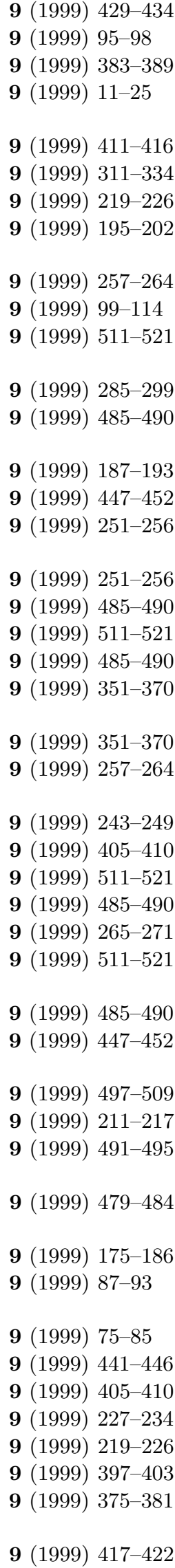


Wu, A. \& Tam, P.K.S., A neural network methodology of quadratic optimization

$\mathrm{Xu}, \mathrm{Y} . \mathrm{Y}$., see $\mathrm{Fu}, \mathrm{H} . \mathrm{-C}$.

Yagi, T., see Kameda, S.

Yeung, D.S., see Shi, D.

Yoneyama, T., see Galvão, R.K.H.

Żak, S.H., see Chan, H.Y.
9 (1999) 87-93
9 (1999) 545-561
9 (1999) 423-428
9 (1999) 497-509
9 (1999) 167-174
9 (1999) 61-74 